\title{
Mycoplasma caviae, a New Species
}

\author{
By AURIOL HILL \\ Medical Research Council Laboratory Animals Centre, Carshalton, Surrey
}

(Accepted for publication 3 December 1970)

So far, three main species of Mycoplasma have been characterized from rodents: Mycoplasma pulmonis, which is associated with chronic respiratory disease in rats and mice suffering from pneumonia and infectious catarrh, $M$. arthritidis, a causative organism of polyarthritis in the rat, and $M$. neurolyticum, which causes the 'rolling syndrome' in mice.

Previously published reports of the isolation of mycoplasmas from guinea pigs (Cavia porcellus) have been few and indefinite (Freundt, I968). Klieneberger (I935) first described the isolation of an organism, now known as Mycoplasma, which was associated with Streptobacillusmoniliformis. Klieneberger also isolated similar organisms from an outbreak of cervical lymphadenitis in guinea pigs (1939). Findlay, Mackenzie \& MacCallum (I940) have described the isolation of similar Mycoplasma-like organisms from their own colony of guinea pigs and from a single animal in another colony. The organisms could not be subcultured, and therefore their identity is uncertain: possibly the colonies were L forms of Streptobacillus moniliformis. This dearth of information regarding Mycoplasma infections in guinea pigs may be due to absence of survey work.

During the course of a general survey and diagnostic autopsies, seven separate Mycoplasma isolates were obtained from guinea pigs (Hill, Blackmore \& Francis, I969). It became evident that the seven different isolates were similar and that they appeared to be dissimilar to the mycoplasmas previously recovered from rodents. It was decided, therefore, to characterize and describe these organisms. The tests performed were those usually employed by workers in this field, and serological comparisons were made with some known rodent and human species.

Source of animals. The six Mycoplasma strains from apparently healthy guinea pigs were isolated during a survey of laboratory animals from accredited breeders. This work was part of the Medical Research Council Laboratory Animals Centre's grading scheme for accreditation (MRC Laboratory Animals Centre, 1969). Eighty-six guinea pigs from 22 colonies were tested. The seventh isolate was obtained post mortem from a guinea pig with metritis from which no other known microbial pathogens were isolated. (Since this paper was prepared there have been six further isolations from guinea pigs.)

Isolation techniques. Brain, ears, nasopharynx, lungs, joints and genital tracts of guinea pigs were examined for the presence of Mycoplasma. Brain cultures were taken by inserting a sterile loop into the cerebrum after the cranium had been deflected. The outer ear was removed to expose the medial part of the external auditory canal, the tympanic membrane was pierced with a pair of sterile forceps, and the middle ear flushed out with Mycoplasma broth using a pasteur pipette. The nasal chamber was 
exposed by cutting through the nose in the mid-maxillary region, and a cotton-wool swab inserted into the exposed turbinate bones. A cut surface of lung was wiped over solid medium and a portion dropped into the liquid medium. The knee joint was clearly exposed by incising the overlying tissues and a swab was taken from the exposed cartilage surface. Cultures were taken of the genital tracts by inserting a swab in either the vagina or prepuce.

Cultural media. The medium used for the isolation of Mycoplasma strains was that used by Taylor-Robinson, Williams \& Haig (1968). To this was added $0.002 \%$ phenol red and $0.1 \%$ glucose, arginine or urea. The solid medium was prepared by the addition of $\mathrm{I}$ g. Oxoid ionagar no. 2 to the basic liquid medium. The plates and broth bottles were incubated aerobically at $37^{\circ}$ for 2 weeks. Growth on methylene blue agar was investigated by adding $0.002 \%$ methylene blue to the basic solid medium.

Biochemical tests. The ability of an isolate to ferment glucose, utilize arginine or split urea was determined using the medium already described and noting any appropriate change in $\mathrm{pH}$. The tetrazolium reduction test was performed by adding $0.0 \mathrm{I} \%$ of 2,3,5-triphenyl tetrazolium chloride to the basic liquid medium. Reduction of tetrazolium was indicated by a deep red colour.

Haemolysis, Haemadsorption and Haemagglutination tests. Haemolytic activity was tested by overlaying a 4 day plate culture with $2 \mathrm{ml}$. of agar medium containing $3 \%$ washed sheep or guinea-pig erythrocytes and reincubating the plates at $37^{\circ}$ for up to 3 days. Haemadsorption and haemagglutination tests were carried out by the method of Manchee \& Taylor-Robinson (1968), using sheep or guinea-pig erythrocytes.

Serological tests. Type species of mycoplasmas were obtained from Dr R. J. Fallon, Dr R. H. Leach, Mr J. T. Heywood and Dr D. Taylor-Robinson. The species were Mycoplasma arthritidis, $M$. canis, $M$. edwardii, $M$. felis, $M$. fermentans, $M$. gallisepticum, $M$. gatae, $M$. hominis, $M$. hyorhinis, $M$. laidlawii, $M$. neurolyticum, $M$. orale, M. pneumoniae and $M$. pulmonis.

Antiserum was produced as described by Morton \& Roberts (I967). A suspension of the organism in $0.1 \mathrm{ml}$ of phosphate-buffered saline and complete Freund's adjuvant was injected into each hind-foot pad of a rabbit, and $0.5 \mathrm{ml}$. intramuscularly into four sites on the shoulders. Three weeks later, two injections of $0.5 \mathrm{ml}$. of the Mycoplasma suspension alone were injected into two sites on the shoulders. The serum was collected after another 7 days.

Growth inhibition tests (Clyde, 1964) were carried out using sterile paper discs impregnated with antisera.

The metabolic inhibition tests were performed as described by Taylor-Robinson, Purcell, Wong \& Channock (I966), using disposable plastic microtitre plates.

Complement-fixation tests were carried out using the microtechnique described by Sever (1962). Antigens were prepared by growing the organisms in $50 \mathrm{ml}$. of liquid Mycoplasma medium for 5 days. The cultures were centrifuged, washed three times with phosphate-buffered saline, and resuspended in I $\mathrm{ml}$. of the buffered solution. Eight units of antigen were used in all complement-fixation tests. Anticomplementary factors were reduced by incubating nine parts of antigen with one part of guinea-pig complement at $37^{\circ}$ for $I \mathrm{~h}$. Antigens and antisera were then heated at $56^{\circ}$ for $30 \mathrm{~min}$. before performing the tests (Taylor-Robinson, Canchola, Fox \& Channock, 1964).

For gel-diffusion tests Mycoplasma suspensions were prepared as already described and treated in an M.S.E. ultrasonic disintegrator at $2 \mathrm{~A}$ for $20 \mathrm{~min}$. The agar used was 
I \% Oxoid ionagar no. 2 in phosphate-buffered saline. The plates had one central and six peripheral wells, and were kept at room temperature.

Fluorescent antibody tests were carried out using Mycoplasma colonies grown for 5 days on solid medium. The colonies were transferred to slides by the water fixation technique of Clark, Fowler \& Brown (I96I). The slides were put on moistened foam rubber in a plastic box. One drop of the appropriate antiserum was placed on the colonies and allowed to react for $30 \mathrm{~min}$. at $37^{\circ}$. The slides were then washed with two changes of phosphate-buffered saline and magnetically stirred for $\mathrm{I} h$. When the slides were dry, antispecies serum conjugated with fluorescin isothiocyanate (NordicFrayburg Ltd, Maidenhead, Berkshire) was applied to the slides, which were incubated as before for $30 \mathrm{~min}$. at $37^{\circ}$. The slides were washed and dried as before, mounted in Uvinert (George T. Gurr Ltd, London) aqueous mountant and viewed by ultraviolet microscopy. A known negative serum and other species of Mycoplasma were included as controls.

All the isolates, except the one connected with metritis, were subcultured four to five times in the glucose broth medium before the organisms could be cultured onto a solid medium. On solid medium, there was visible growth in 3 to 5 days after inoculating the plates. All the isolates apparently need sterol for growth, as they were unable to grow on nutrient agar.

\section{Biochemical activity}

All the isolates fermented glucose within 5 days and did not utilize arginine or split urea. They grew weakly in the presence of methylene blue and did not reduce tetrazolium chloride.

The isolates produced haemolysis with sheep and guinea-pig erythrocytes after $24 \mathrm{~h}$. incubation. No haemadsorption was observed with any of the isolates using sheep and guinea-pig erythrocytes. The isolates did not agglutinate sheep or guinea-pig erythrocytes.

\section{Serological test results}

Antisera prepared against each specific strain of the guinea-pig mycoplasmas inhibited the growth of all the other strains isolated from guinea pigs to a comparable degree. Other species of Mycoplasma were not inhibited by these guinea-pig antisera except for a very slight inhibition of Mycoplasma neurolyticum and $M$. pneumoniae. Metabolic inhibition tests showed that the guinea-pig Mycoplasma strains were inhibited by all the antisera against these different isolates to a titre of $1 / 320$ to $\mathrm{I} / 640$. Mycoplasma fermentans, $M$. neurolyticum amd $M$. pulmonis were inhibited to a titre of $1 / 20$, whilst all other species of Mycoplasma showed no evidence of inhibition. Complementfixation tests, in which the homologous antigen reacted to a level of $I / 800$, showed no cross-reaction above a level of $1 / 50$ between one of the strains isolated from guinea pigs and the other known species of Mycoplasma examined.

Gel-diffusion tests showed a strong reaction between all the guinea-pig isolates, and a slight cross-reaction between the guinea-pig isolates and Mycoplasma neurolyticum.

All the guinea-pig Mycoplasma colonies treated with the antisera to any of the other guinea-pig Mycoplasma isolates showed obvious specific fluorescence, while control specimens treated with antisera specific for other recognized mycoplasmas failed to produce a positive reaction. 
The results recorded in this paper unequivocally demonstrate that on seven separate occasions Mycoplasma species have been isolated from guinea pigs, and all the isolates appeared to be similar. Although Mycoplasma species usually exhibit a degree of host specificity, instances are increasing of organisms which are usually associated with a particular host species but which can be recovered from an unrelated species of animal, e.g. Mycoplasma gallinarum and $M$. iners from pigs (Taylor-Robinson \& Dinter, 1968) and $M$. arthritidis from man (Edward \& Freundt, 1965). The guinea-pig isolates described in this report fermented glucose and did not reduce tetrazolium chloride. They showed no significant serological relationship with any known Mycoplasma species having these characteristics, nor was there any cross-reaction with Mycoplasma species showing different biochemical reactions against which they were tested. These results indicate that the guinea-pig isolates are different from any other known species of Mycoplasma. It is suggested that this apparently unrecorded new species of Mycoplasma should be called Mycoplasma caviae.

The infected animals were from seven different colonies out of a total of 23 examined. Although the total number of animal colonies examined was rather small, approximately $30 \%$ of the colonies were infected. This suggests that Mycoplasma infection, or carriage, in guinea pigs may be relatively high. The lack of other reports of this type of infection could be due to a variety of reasons. As only one guinea pig in each batch of four from colonies examined was affected, the organism may only occur in relatively few animals within the colony; also, the organism was not confined to a specific site, being isolated from the nasopharynx in four cases, the genital tract in two, and the brain in one. In all but one case, the infected animals were clinically normal, so that unless specific cultures were put up during autopsy, the organism could easily be missed. Another possible reason for the lack of previous records may be seen in the difficulty of isolating this species, which, unlike other rodent mycoplasmas, has to be subcultured several times through an enriched liquid medium before being successfully cultured on a solid medium.

It is not yet known whether this guinea-pig Mycoplasma species is pathogenic, but as one of the strains from guinea pigs was associated with a uterine infection, Mycoplasma caviae could be a potential pathogen. Transmission studies are being carried out to obtain further information.

The author wishes to thank Dr D. G. ff. Edward for his advice, and Dr D. K. Blackmore for help in preparing this paper.

\section{REFERENCES}

Clark, H. W., Fowler, R. C. \& Brown, T. M. (196I). Preparation of pneumonia-like organisms for microscopic study. Journal of Bacteriology 81, 50I-502.

CLYDE, W. (I964). Mycoplasma species identification based upon growth inhibition by specific antisera. Journal of Immunology 92, 958-965.

Edward, D. G. ff. \& Freundt, E. A. (1965). A note on the taxonomic status of strains like 'Campo', hitherto classified as Mycoplasma hominis, type 2. Journal of General Microbiology 4r, 263-265.

Findlay, G. M., Mackenzie, R. D. \& MacCallum, F O. (I940). Chemotherapeutic experiments on pleuropneumonia-like organisms in rodents. British Journal of Experimental Pathology 2r, 1322.

FREUNDT, E. A. (1968). Mycoplasma infections in laboratory rodents. Zeitschrift für Versuchstierkunde I0, I 7-26. 
Hill, A. C., Blackmore, D. K. \& Francis, R. A. (1969). The isolation of mycoplasmas from guinea pigs (Cavia porcellus). The Veterinary Record 8r, 29r-292.

KLIENEBERGER, E. (1935). The natural occurrence of pleuropneumonia-like organisms in apparent symbiosis with Streptobacillus moniliformis and other bacteria. Journal of Pathology and Bacterio$\log y$ 40, 93-105.

KIIENEBERGER, E. (1939). Studies on pleuropneumonia-like organisms. Bacteriological features and serological relationship of strains from various sources. Journal of Pathology and Bacteriology 49, $451-452$.

MANCHEE, R. J. \& TAYLOR-RoBinson, D. (I968). Haemadsorption and haemagglutination by mycoplasmas. Journal of General Microbiology 50, 465-478.

MRC LABORATORY ANIMALS CENTRE (1969). The Accreditation and Recognition Schemes for Suppliers of Laboratory Animals. Carshalton, Surrey: MRC Laboratory Animals Centre.

Morton, H. E. \& RoberTs, R. J. (1967). Production of antimycoplasma (PPLO) antibodies in rabbits. Proceedings of the Society for Experimental Biology and Medicine 125, 538-543.

SeVER, J. L. (1962). Application of a microtechnique to viral serological investigations. Journal of Immunology 88, 320-329.

Taylor-Robinson, D., Canchola, J., Fox, J. \& Channock, R. M. (1964). A newly identified oral Mycoplasma ( $M$. orale) and its relationship to other human mycoplasmas. American Journal of Hygiene 80, 135-148.

TAYLOR-RoBINSON, D. \& DINTER, Z. (I968). Unexpected serotypes of mycoplasmas isolated from pigs. Journal of General Microbiology 53, 22 I-229.

Taylor-Robinson, D., Purcell, R. H., Wong, D. C. \& Channock, R. M. (1966). A colour test for the measurement of antibody to certain Mycoplasma species based upon the inhibition of acid production. Journal of Hygiene 64, 9I-104.

Taylor-Robinson, D., Williams, M. H. \& HaIG, D. D. (I968). The isolation and comparative biological and physical characteristics of T-mycoplasmas of cattle. Journal of General Microbiology 54, 33-46. 